\title{
Balkanologie
}

Balkanologie Revue d'études pluridisciplinaires

Vol. VIII, $n^{\circ} 1 \mid 2004$

Volume VIII Numéro 1

\section{Présentation de soi et narration de l'histoire}

Les enjeux de la transformation d'un mémorial de la Seconde Guerre mondiale en Serbie

Self-presentation an narration of history. The stakes in the transformation of a Second World War memorial in Serbia

Dejan Dimitrijevic

\section{(2) OpenEdition}

\section{Journals}

Édition électronique

URL : http://journals.openedition.org/balkanologie/524

DOI : 10.4000/balkanologie.524

ISSN : 1965-0582

Éditeur

Association française d'études sur les Balkans (Afebalk)

Édition imprimée

Date de publication : 1 juin 2004

ISSN : 1279-7952

\section{Référence électronique}

Dejan Dimitrijevic, « Présentation de soi et narration de l'histoire », Balkanologie [En ligne], Vol. VIII, $n^{\circ} 1$ । 2004, mis en ligne le 21 janvier 2010, consulté le 17 décembre 2020. URL : http://

journals.openedition.org/balkanologie/524 ; DOI : https://doi.org/10.4000/balkanologie.524

(c) Tous droits réservés 


\section{PRÉSENTATION DE SOI ET NARRATION DE L'HISTOIRE. LES ENJEUX DE LA TRANSFORMATION D'UN MÉMORIAL DE LA SECONDE GUERRE MONDIALE EN SERBIE.}

Dejan Dimitrijevic*

\section{LES ANNÉES 1980 : RETOUR EN FORCE DU PASSÉ CENSURÉ}

La mort du Maréchal Tito, en 1980, ouvre une décennie qui a vu émerger toutes les contradictions et tous les antagonismes de la société yougoslave, car le passé y a fait une entrée en force, notamment celui relatif à la Seconde Guerre mondiale. L'histoire officielle yougoslave présentait une vision schématique de cette période, en gommant toutes les aspérités et en réduisant toute la complexité sociale et historique. Il y avait d'un côté la Résistance, plurinationale et progressiste, dirigée par le Parti communiste yougoslave, avec à sa tête Josip Broz Tito, et de l'autre les forces réactionnaires nationales, principalement les Oustachis croates, avec une composante bosniaque musulmane, et les Tchetniks serbes. Cette histoire officielle était en bonne partie en contradiction avec les mémoires collectives nationales et familiales. Nous nous intéresserons ici tout particulièrement au cas de la Serbie.

Avant de sombrer dans le nationalisme, dans la seconde moitié des années 1980, la société yougoslave se caractérisait par une relative démocratisation politique qui a permis l'expression publique de la mémoire collective en Serbie, comme dans les autres Républiques yougoslaves. L'apparition dans l'espace public des mémoires collectives nationales et familiales, qui ont le plus souvent pour source le récit autobiographique d'un parent, était un phénomène nouveau en ex-Yougoslavie, car ces mémoires n'étaient jusqu'alors présentes que dans la sphère privée. Le passage de la sphère privée à la sphère publique est parfaitement illustré par l'immense succès du Roman de Danko 
Popovic, Le livre de Miloutine ${ }^{1}$, publié en 1986. Le traducteur français en fait un résumé très précis sur sa quatrième de couverture.

Miloutine est vieux, Miloutine est en prison, Miloutine va mourir - et il parle. Il parle à en perdre le souffle, il veut raconter sa vie avant qu'il ne soit trop tard, parce qu'il est à lui tout seul la mémoire de sa génération.

Petit paysan de la Serbie du Sud ${ }^{2}$, il a traversé notre siècle en faisant une guerre 3 , deux guerres 4 , et encore une, puis une autre, quatre en tout, auxquelles il n'aura pas compris grand chose, sinon qu'on avait besoin de sa force, de son courage, de son sang, et qu'il fallait bien y aller.

Qui se souvient encore de la Serbie ? Partie intégrante de cette Yougoslavie qu'elle a contribué à édifier au prix de tant de souffrances, elle a caché ses deuils et ses blessures - jusqu'à ce que les événements récents du Kosovo la fassent ressurgir de façon inquiétante des profondeurs de l'oubli. Miloutine, lui, a tout connu : la lutte contre les Austro-Hongrois en 1914, puis la retraite d'hiver vers l'Albanie, puis la guerre en Afrique et à Salonique. Un million deux cents mille Serbes auront péri... éclate la Seconde Guerre mondiale et Miloutine repart se battre contre les Allemands cette fois, et après cinq ans d'enfer, à la fin des combats, on le jette en prison parce qu'il a un lopin de terre, il est riche, c'est un " koulak "...

Alors vite, il raconte, dans sa langue simple et savoureuse, avec une belle lucidité et une résignation pleine de fierté, les péripéties de son humble vie. Il est un de ces êtres honnêtes, sans nom, sans visage qui, par leurs sacrifices tissent la trame de l'Histoire. Ce livre exceptionnel, traduit dans le monde entier, s'est vendu en Yougoslavie seule à quatre cent mille exemplaires et a été couronné par le plus grand prix littéraire du pays.

Incarnation du peuple serbe, la vie de Miloutine permet à la mémoire collective serbe de sortir de la sphère domestique par l'intermédiaire d'une autobiographie fictive. Le Livre de Miloutine restitue l'état d'esprit de la population serbe des années 1980 et ses interrogations sur le sens de l'histoire yougoslave, à un moment où l'État yougoslave, plongé dans une grave crise économique, sociale et politique, ne semble plus en mesure de porter un projet d'avenir.

Le récit de Miloutine est en réalité une mise en cause de l'histoire officielle yougoslave. Les Partisans communistes n'ont plus le beau rôle : le roman met en scène leur rejet par le " peuple paysan serbe ", qui est le véritable héros du livre. Mais si les Partisans deviennent des héros négativement connotés, le Mouvement tchetnik n'est pas encore dépeint positivement : les uns et les autres sont renvoyés dos-à-dos mais progressivement, l'évocation publique des

\footnotetext{
${ }^{1}$ Le roman a été traduit en français chez Stock, Nouveau Cabinet Cosmopolite, en 1989. Dans sa version originale, Knjiga o Milutinu a été publié par Književne Novine à Belgrade.

${ }^{2}$ La Šumadija (prononcez Shoumadiya) se trouve, en réalité, en Serbie centrale.

${ }^{3}$ Première Guerre balkanique (1912).

4 Deuxième Guerre balkanique (1913).
} 
Tchetniks n'est plus interdite ; et l'introduction du multipartisme rend possible la constitution, en 1990, d'un parti politique, le SPO5, dirigé par Vuk Drašković, qui œuvre à la réhabilitation du Mouvement tchetnik et s'inscrit dans une filiation idéologique qui s'en revendique. Il a été aussitôt utilisé par le Parti socialiste serbe ${ }^{6}$ comme repoussoir électoral, en le présentant comme extrémiste, tout en développant un discours dont les thèmes principaux sont : les intérêts de la République socialiste de Serbie 7 , mis à mal par la Constitution de 1974, les victimes serbes des Oustachis croates et musulmans de la Seconde Guerre mondiale, et les droits des Serbes dans les autres républiques de la Fédération.

Les forces nationalistes ont exploité à outrance les frustrations de la période titiste, et particulièrement celles liées à la mémoire de la Seconde Guerre mondiale. Nous avons encore en tête les images des victimes serbes des massacres oustachis qui étaient régulièrement projetées à la télévision serbe à la fin des années 1980 et au début des années 1990. Désormais la production culturelle serbe est dominée par la mise à l'ordre du jour du passé serbe, qui est le plus souvent présenté comme glorieux, dans la victoire comme dans la défaite.

L'année 1987 a été déterminante pour un tel développement politique et culturel. Yves Tomić, dans son article " La ligue des communistes de Serbie et l'ouverture de la question nationale serbe : 1977-1987 " ${ }^{8}$ montre très bien comment Milošević remet la bataille du Kosovo de 1389 au centre de la vie politique serbe, ce qui n'était plus le cas depuis le XIX ${ }^{\mathrm{e}}$ siècle$^{9}$. L'histoire devient un des principaux arguments politique serbe, et le lien culturel le plus solide durant toutes les années de guerre des années 1990 et jusqu'à la chute du régime de Milošević. Le passé était si présent que les Serbes ont pu apparaître comme un peuple obsédé par l'histoire.

Après le renversement du régime de Milošević, la politique culturelle serbe, et plus particulièrement le traitement de son passé, tentent d'emprunter une autre direction. Examinons les nouvelles orientations au travers d'un exemple significatif et emblématique : Le Musée-mémorial " 21 Octobre " de Kragujevac ${ }^{10}$.

\footnotetext{
5 Srpski Pokret Obnove (Mouvement serbe du renouveau).

${ }^{6}$ Nouveau nom du Parti communiste serbe.

${ }^{7}$ La critique principale porte sur l'éclatement de la République socialiste de Serbie en trois partie : la Voïvodine, le Kosovo et la Serbie ètroite.
}

${ }^{8}$ Tomic (Yves), « La ligue des communistes de Serbie et l'ouverture de la question nationale serbe : 19771987 ", Balkanologie, 3 (1), septembre 1999.

9 À ce propos voir aussi Cerovic (Stanko), Dans les griffes des humanistes, Castelnau-le-Lez : Sysiphe/Climats, 2001 [1999].

${ }^{10} \mathrm{Il}$ s'agit d'un terrain en cours d'exploration. 


\section{LES MASSACRES ALLEMANDS D'OCTOBRE 1941 ET LES ENJEUX DU MEMORIAL DE KRAGUJEVAC}

Après la victoire allemande en avril 1941, deux mouvements de résistance se constituent sur le territoire serbe, et toutes deux ont pour premier terrain d'action la Šumadija, en Serbie centrale. L'une est dirigée par le Parti communiste, et l'autre par des cadres de l'armée yougoslave qui décident de continuer la lutte au nom du Roi. Les Partisans et les Tchetniks unissent leurs forces, dans un premier temps, avant de devenir les pires ennemis. Pendant cette brève période de coopération, d'assistance militaire et de non agression, la résistance unifiée connaît un certain succès et libère un certain nombre de villes, dont Gornji Milanovac le 29 septembre 1941. En représailles, les forces allemandes prennent en otage plusieurs milliers de serbes et de juifs de Kragujevac et des villages environnants.

Et du 19 au 21 octobre, plusieurs milliers d'otages, dont des femmes et des enfants, sont fusillés à Kragujevac, dans un lieu appelé Šumarica. Le nombre exact des victimes n'est pas connu : le musée de Kragujevac possède des documents sur 2246 fusillés et 29 rescapés; les sources allemandes parlent de 2300 tués, et la Commission d'État des crimes de guerre, dans son rapport du 15 juillet 1945, avance le chiffre de 2323 tués. Cependant, au procès de Nürenberg le nombre de victimes retenu était de $7000^{11}$.

Dans les années 1970, un mémorial est construit sur le lieu du massacre, sur 352 hectares, et le musée est inauguré le 15 février 1976. Le musée et le mémorial étaient mis au service idéologique du pouvoir communiste. L'exposition permanente se composait de l'historique de Parti communiste yougoslave, et de trois photographies de trois héros nationaux : deux communistes notoires (un homme et une femme) et un directeur d'école, également présenté comme communiste, mais dont tout le monde savait qu'il n'avait aucune sympathie pour les Partisans, selon les actuels responsables du musée ${ }^{12}$.

Le message de ce mémorial, qui était pensé en culte politique, se serait probablement éteint, comme tous les monuments aux morts avec la disparition des survivants et l'affaiblissement de l'idéologie qu'il représentait. C'est le destin de ce type de monuments nous dit R. Koselleck ${ }^{13}$ mais l'enjeu historique qu'il constitue pour la période actuelle l'a transformé en un lieu expérimental pour la mise en place d'un nouvel équilibre idéologique, national et international.

${ }^{11}$ Ce sont les chiffres présentés dans la brochure du musée.

${ }^{12}$ Nenad Djordjević, directeur, et Staniša Brkić, conservateur en chef.

${ }^{13}$ Koselleck (Reinhart), L'expérience de l'histoire. Les monuments aux morts, lieux de fondation de l'identité des survivants, Paris : EHESS/Gallimard/Le Seuil, 1997, pp. 135-160. 
Dans le cadre de ce mémorial doit s'ouvrir un Centre pour la paix et la tolérance. "Le changement de concept ", nous dit le conservateur en chef Staniša Brkić ${ }^{14}$, " va se mettre en place sans oublier les fondements, le mémorial aux victimes, mais il sera conçu comme une ouverture vers la paix et l'échange, ici et avec le reste du monde ". Impulsé par le Ministère de la culture, et financé en bonne partie par USAID, le Centre doit favoriser le " rapprochement des peuples et des cultures à travers la culture ". Le nouveau concept bénéficie de soutiens ministériels et institutionnels serbes et d'un financement international. Ainsi le ministère de la culture serbe, le musée d'histoire de Serbie et le Conseil municipal de Kragujevac favorisent la réalisation du projet, alors que le budget provient principalement d'organisations telles que USAID, le Ministère de la culture allemand, et d'autres liées à l'Union Européenne et à l'ONU.

La première manifestation du nouveau concept s'est déroulée en 2002, sous la forme d'une exposition de peinture regroupant 40 peintres des pays de l'ex-Yougoslavie ${ }^{15}$ - dont un peintre albanais du Kosovo - des pays balkaniques et d'Europe occidentale. Cette exposition, intitulée "Les ponts des Balkans ", a été interrompue pour cause de montage de la nouvelle exposition permanente, et elle doit reprendre en 2004.

La nouvelle exposition permanente devait officiellement remplacer celle de 1976, qualifiée d'idéologique, le 15 février 2003, mais les travaux ayant pris du retard, son inauguration était reprogrammée pour la fin de l'année 2003 . Le conservateur en chef la présentait comme " historiquement très propre, sans idéologie aucune, elle sera réalisée avec les moyens technologiques actuels ".

Mais le nouveau concept concerne également le parc qui abrite 30 tombes collectives, dont 10 possèdent des monuments funéraires de grande taille, qui sont des œuvres importantes. Et s'il n'est pas question de toucher à ces sculptures, les plaques sur les tombes doivent être remplacées.

"Les plaques des tombes seront changées. C'était le signe d'un temps passé qui n'a plus de raison d'être. Nous enlèverons les signes communistes et les textes. Nous avons déjà enlevé une plaque qui indiquait : "Ici ont été fusillés les plus progressistes de nos fils" "16. La volonté d'effacer l'appropriation idéologique des victimes s'accompagne aussi du désir de mettre fin à l'appropriation exclusive communiste de la lutte contre le fascisme et le nazisme. " Le musée doit aussi montrer l'autre face de l'histoire de la Seconde Guerre mondiale. Il doit rétablir la vérité sur les Tchetniks ". Cette réhabilitation a déjà commencé puisque dans la brochure actuelle du mémorial, nous voyons une

\footnotetext{
14 L'entretien s'est déroulé en novembre 2002.
}

15 Seule la Slovénie n'était pas représentée.

16 Staniša Brkić. 
photographie d'un très important groupe de prisonniers allemands encadré par les Tchetniks ; ce qui signifie clairement que ces derniers n'étaient pas des collaborateurs, mais bien des résistants ; en tout cas ni plus collaborateurs ni moins résistants que les Partisans.

Le directeur du mémorial, Nenad Djordjević, confirme que le nouveau concept est construit autour des valeurs de " tolérance ", de " réconciliation ", et dans un " esprit pacifiste ", qui doit englober le " passé, celui de la Seconde Guerre mondiale, la période actuelle et la construction du futur ". Ce projet, dont la réalisation a déjà commencé, est encouragé par les Institutions internationales pour favoriser la normalisation entre les pays issus de la Fédération yougoslave et l'écriture d'une histoire qui ne soit plus une ressource pour des conflits futurs. Pour réaliser la triple réconciliation des Serbes avec les puissances occidentales, avec les voisins de l'ex-Yougoslavie (Croates, Bosniaques musulmans et Albanais) et des Serbes entre eux, il semblerait qu'il faille, pour le moins, neutraliser l'histoire. Pour comprendre cette nouvelle relation au passé, il est nécessaire de replacer ce phénomène dans la configuration idéologique actuellement dominante, qui propose une révision de la place du passé.

\section{PASSÉ ET PACIFICATION : QUELLE RELATION AUJOURD'HUI ?}

L'idée qu'il faut se débarrasser de l'histoire pour prévenir les conflits se répand depuis quelque temps. Au sujet de la " transition " de l'Europe de l'Est, et encore plus au sujet des guerres dans l'ex-Yougoslavie, un grand nombre d'" experts " occidentaux préconisent l'oubli du passé car " qui se souvient de l'histoire est condamné à la répéter ${ }^{17}$. Même la poursuite de la vérité et de la justice est perçue comme dangereuse et vaine, car elle serait en conflit avec la nécessité de réconciliation et la voie ouverte à une nouvelle guerre puisqu'il y a peu de chances pour que toutes les parties reconnaissent la même vérité ${ }^{8}$. Le développement des échanges économiques est présenté comme le lien intra-groupes et inter-groupes suprême ${ }^{19}$. Et ce sont les États-Unis d'Amérique qui servent de modèle, selon l'idée que la grande force culturelle des États-Unis réside dans sa capacité à se débarrasser du passé en redémarrant une nouvelle histoire ${ }^{20}$.

\footnotetext{
${ }^{17}$ Voir Offe (Claus), “ Ethnic politics in East European Transitions ", Diskussionspapier, (1), 1993, pp.22-23.

${ }^{18}$ Ignatieff (Mickael), "Articles of Faith ", Index on Censorship, (5), 1996, cité d'après Jedlicki (Jerzy), " Historical memory as source of conflicts in Eastern Europe ", Communist and Post-Communist Studies, 32, 1999, p. 231.

${ }^{19} \mathrm{M}$. Kaminski préconise la réconciliation des ennemis en Bosnie-Herzégovine par l'oubli du passé et le développement des échanges économiques (Kaminski (M.), " Czy handel zjednoczy dotychczaswych wrogow ? " (Le marché va-t-il unir les ennemis ?), Wall Street Journal Europe (supplément de la Gazeta Wyborcza), 26/10/98).
}

${ }^{20}$ Jedlicki (Jerzy), art.cit., p. 231. 
Ce rapport au passé apparaît comme lié à des phénomènes idéologiques contemporains. Pour Giovanni Levi la dévalorisation du passé est à mettre en rapport avec les révisionnismes d'aujourd'hui, qui n'ont plus pour objectif de réévaluer des mouvements, des personnages ou des périodes, mais de dévaluer le passé en le chargeant de laideurs. Ce rapport au passé, affirme G. Levi, accompagne l'annonce de la fin des idéologies et ouvre la voie à l'irrationalisme, au néo-libéralisme, au nationalisme, au fondamentalisme religieux. Les significations se mélangent et les fautes se font égales. Cette vision de l'histoire donne une image du passé qui déborde en général de mal sans que l'on puisse en identifier avec précision une source, si ce n'est de manière abstraite ${ }^{21}$.

Jérôme Baschet caractérise cette tendance à étouffer la connaissance critique du passé, et qui s'accompagne d'un obscurcissement des perspectives du futur, comme la domination d'un présent perpétuel qui s'impose avec l'annonce de la fin de l'histoire. L'oubli et la négation du futur sont alors analysés comme des traits caractéristiques du néo-libéralisme : " Dans le monde moderne en voie de globalisation, l'aujourd'hui est le nouveau tyran qui, pour mieux assurer sa domination, fait sombrer le passé dans l'oubli et obnubile toute perspective d'un futur qui ne soit pas la répétition ou l'amplification de la domination présente $n^{22}$.

Le post-modernisme semble être une autre manifestation de la même logique. Ne reconnaissant aucune possibilité de synthèse et décrétant que l'universel est une illusion, le post-modernisme pousse le relativisme à l'extrême : il annonce le règne du particularisme et de la subjectivité, et n'accorde aucun crédit à la recherche de la vérité. De facto, l'Histoire universelle devient impossible ${ }^{23}$. C'est ce que Régine Robin appelle un " révisionnisme généralisé ", porteur " d'un nivellement relativiste ", où nazisme, stalinisme, guerres coloniales... sont renvoyés dos à dos. Elle illustre ce phénomène, entre autres, par le nouveau récit du Karlshorst Museum de Berlin, qui est le lieu de la reddition sans condition de l'Allemagne le 8 mai 1945.

Autrefois, comme c'était en zone soviétique et en RDA après la fondation de l'Etat, le musée était à la gloire de la grande guerre patriotique de l'URSS, à la gloire de la prise de Berlin. Tout alors était clair, idéologique, directement idéologique. Nos historiens ont complètement revu le discours. C'était beaucoup trop idéologique pour eux, surtout après les événements de 1989 et la réunification. (...) Tout dans le discours participe du grand nivellement. Dans le fond, on est passé de la grande

\footnotetext{
${ }^{21}$ Levi (Giovanni), " Le passé lointain ", in Hartog (François), Revel (Jacques), éds., Les Usages politiques du passé, Paris : EHESS (coll. Enquête, (1)), 2001, pp. 35-37.

${ }^{22}$ Baschet (Jérôme), " L'histoire face au présent perpétuel n, in Hartog (François), Revel (Jacques), éds., op.cit., p. 63.

${ }^{23}$ Wachtel (Andrew), "Kada i zašto je "jugoslovenska kultura" imala smisla " (Quand et pourquoi la " culture yougoslave » avait du sens), Sarajevske sveske, (1), 2002.
} 
guerre patriotique, de la grande Armée rouge qui avait pris Berlin, à un discours que je résumerai en une phrase : à Stalingrad, il faisait froid pour tout le monde. ${ }^{24}$

Comme en écho à ce constat, Gérard Lenclud nous dit que le seul passé désormais estimable est celui dont on a le sentiment qu'il est parfaitement maîtrisé ${ }^{25}$. Le passé serait, aux yeux des contemporains, comme un volcan éteint, on s'y promène sans danger.

Le passé est donc bien souvent regardé comme éminemment suspect ; objet de méfiance, il est présenté comme cause au moins potentielle de désordres et de conflits. De plus en plus nombreux sont ceux qui, à l'instar de Paul Ricœur ${ }^{26}$, plaident pour un " art d'oublier ", afin d'en finir avec les " pathologies de la mémoire " et une société " éternellement en colère avec elle même ". Il est vrai que pour continuer, une société ne peut pas être indéfiniment en colère avec elle-même. Dans ces conditions elle peut encore moins se construire. C'est la raison pour laquelle, dans le cadre de la construction européenne, nous retrouvons dans la bouche ou sous la plume de certains responsables politiques des paroles dignes du préambule de l'édit de Nantes qui décrétait " que la mémoire de toutes choses passées d'une part et d'autre demeurera éteinte et assoupie comme des choses non advenues ". En 2003, Romano Prodi, Président de la Commission Européenne, également soucieux de réconciliation et d'unité, déclare aux responsables politiques croates, lorsque la Croatie fait officiellement acte de candidature d'adhésion que " adhérer à l'Union européenne, c'est changer sa propre histoire : l'UE est une véritable union, et non une zone d'intégration purement technique et économique ${ }^{27}$. Faut-il souligner qu'en la matière, les décrets n'ont pas montré leur efficacité : les affrontements militaires entre catholiques et protestants ont repris après l'assassinat d'Henri IV en 1610.

Dans ces conditions, quelle mémoire peut se constituer ? Il n'est pas certain que la mémoire qui se met en scène actuellement dans les musées de " réconciliation " puisse encore être appréhendée par l'herméneutique classique de l'anthropologie ou de la sociologie de la mémoire développées à la suite de Maurice Halbwachs ; celle qui reconnaît une mémoire collective qui reconstruit le passé.

Cette nouvelle logique mémorielle semble beaucoup plus proche de la philosophie morale pour qui seuls comptent, moralement, les vivants, non les morts.

\footnotetext{
${ }^{24}$ Robin (Régine), " Une juste mémoire, est-ce possible ? ", in Ferenczi (Thomas), Devoir de mémoire, droit à l'oubli ?, Paris : Complexe, 2002, p. 111.

${ }^{25}$ Lenclud (Gérard), "L'usine au musée ou le passé consommé. Octave Debary, La Fin du Creusot ou l'Art d'accomoder les restes ", Critique, décembre 2003.
}

${ }^{26}$ Ricœur (Paul), "Esquisse d'un parcours de l'oubli ", in Ferenczi (Thomas), op.cit.

${ }^{27}$ Le Monde, 25/02/03, p. 6. 
Certes, nous dit Marcel Conche, la philosophie morale n'exclut pas que l'on se souvienne des morts, mais elle n'en fait pas une obligation (c'est à chacun, dans l'intimité de son amour, de se souvenir de ses morts). La mémoire qui lui importe n'est pas la mémoire du passé, mais la mémoire du présent. Ce dont on doit se souvenir, c'est de ce qui se passe ou a lieu présentement dans le monde, et qui a, chaque fois, le caractère d'un événement absolu, bon ou mauvais en soi. ${ }^{28}$

Cette logique morale se focalise sur le présent et sur l'universel. Nous reconnaissons là, dans sa dimension déclarative, les valeurs fondamentales de l'humanisme mises en avant par les grandes démocraties occidentales. Mais un examen rapide de l'actualité internationale révèle immédiatement que la dynamique idéologique dominante n'est pas véritablement celle de la philosophie morale qui pose comme principe fondamental que tout événement qui constitue une offense à la dignité de l'homme a le caractère d'événement absolu. Entre les principes déclaratifs et les pratiques des puissances économiques et politiques européennes et nord-américaines le décalage semble parfois incommensurable. Cela n'échappe à personne ; mais pour obtenir une place dans les institutions internationales et exister économiquement et politiquement, les " petits pays " doivent inscrire la présentation de soi dans le cadre du grand discours fondateur du monde libéral contemporain présenté comme l'aboutissement de l'histoire, et qui est sous-tendu par les maître-mots d'ouverture et de tolérance.

\section{LA CULLTURE, ENTRE DÉSIR UNIVERSALISTE ET REALITE NATIONALE}

Après le renversement du régime de Milošević, la politique culturelle serbe, et plus particulièrement le traitement de son passé, ont été fortement influencées par l'Union européenne et les États-Unis. Après le processus de fermeture des frontières identitaires des années 1990, nous assistons actuellement à une ouverture de ces frontières pour permettre l'adaptation des pays issus de l'exYougoslavie au contexte idéologique dominant à l'échelle mondiale et leur intégration dans un grand ensemble significatif à cette échelle, l'Union européenne. C'est ce processus qui se laisse percevoir au travers de la transformation du mémorial " 21 Octobre ". Il implique non seulement des transformations politiques et économiques, mais aussi identitaires et culturelles. Ce sont la présentation de soi et l'autocompréhension ${ }^{29}$ qu'il s'agit de transformer car l'injonction extérieure exprimée explicitement par Romano Prodi est suffisamment claire ; et ce

${ }^{28}$ Conche (Marcel), Le fondement de la morale, Paris : PUF, 1999 (1993), p. 136.

29 Brubacker (Rogers), “ Au-delà de l'identité ", Actes de la recherches en sciences sociales, (139), septembre 2001. 
qui est valable pour la Croatie, l'est encore plus pour la Serbie. Dans ce processus d'intégration au monde global, le gouvernement serbe tente de mettre en place une politique qui corresponde aux canons internationaux.

Le nouveau concept du mémorial ne correspond pas seulement à des exigences extérieures, le " rapprochement des peuples et des cultures à travers la culture " est également une vision de la culture partagée par des organisations non gouvernementales et des médias indépendants du pouvoir des années 1990 , et qui étaient proches du $\mathrm{DOS}^{30}$ ou actifs en son sein. En opposition à l'ambiance dominante de la décennie précédente, qui enfermait la culture dans le national, des intellectuels engagés en politique comme Dragoljub Mičunović proposaient un " modèle de la culture cosmopolite, universaliste, comme fondement de l'humanité $"^{31}$. Dans cette logique, le passé serbe doit participer à la formation du modèle narratif de cet humanisme nouveau qui devient explicite à chaque intervention militaro-humanitaire, mais que nous voyons aussi à l'œuvre dans le processus de construction de l'identité européenne du XXI ${ }^{\mathrm{e}}$ siècle. La transformation du Mémorial " 21 Octobre " relève de cette logique, et la forme est respectée à la lettre, mais à y regarder de plus près, on s'aperçoit que ce n'est pas à ce niveau du message que se situe le principal enjeu. À l'arrière plan du cadre narratif de la " paix et de la tolérance " dans le rapport aux autres se joue la réconciliation des Serbes entre-eux et avec leur propre histoire. Et ce n'est pas le moindre des paradoxes que de voir les puissances occidentales imposer un cadre narratif qui est utilisé pour réhabiliter ces Tchetniks devenus synonymes, dans les années 1990, de mal absolu.

La transformation du Mémorial " 21 octobre " révèle un " grand détour " qui passe par le message universaliste globalisant de la paix et de la tolérance pour mieux revenir à soi et à ses conflits internes non résolus.

La transformation du Mémorial " 21 Octobre " en Centre pour la Paix et la tolérance est en passe de se réaliser. Mais pour qui ? Les mécontentements locaux sont importants : entre les accusations de révisionnisme des uns, et celles de perpétuation de la " vérité " communiste des autres, qui lui reprochent de ne pas procéder à une inversion totale de perspective, le Centre pour la paix et la tolérance n'aura pas une tâche facile. La société serbe étant encore en bonne partie structurée par l'histoire de la Seconde Guerre mondiale. Le Mémorial " 21 Octobre " souligne le fait que l'enjeu le plus profond et le plus problématique pour la société serbe est la réconciliation entre Serbes. Ainsi, une entreprise se présentant comme totalement dépolitisée englobe en réalité une des plus délicates dimensions de la vie politique serbe.

${ }^{30}$ Demokratska Opozicija Srbije (Opposition démocratique de Serbie) : coalition de quelque 18 partis politiques et associations qui a contraint Slobodan Milošević a la démission suite à la chute de son régime le 5 octobre 2000 .

${ }^{31}$ Čolović (Ivan), “ Kultura, nacija, teritorija " (Culture, nation, territoire), Republika, (288-289), juillet 2002. 